Forschende

Komplementärmedizin und Klassische Naturheilkunde

\section{Tagungen und Kongresse $\cdot$ Meetings and Conferences}

Forsch Komplementärmed Klass Naturheilkd 2003;10:114

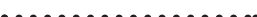

14.05.-17.05.2003

Driebergen

Niederlande

\section{September 2003}

Überlingen

Deutschlanc

[................

$02.10 .-08.10 .2003$

Freudenstadt

Deutschland

\section{$07.11 .-09.11 .2003$}

Bremen

Deutschland

\subsubsection{3}

Solothurn

Schweiz

\subsection{1.-30.11.2003}

München

Deutschland
Congress at the Louis Bolk Institute in the series: "Such is life!"

\section{Summer School Bodensee-Konferenz}

105. ZÄN-Kongress

Bremer-Chiropraktik-Semina

Thema: LWS-Becken

18. Schweizerische Tagung für Phytotherapie - Phytotherapie und Stoffwechsel

ZÄN-Adventskongress
Auskunft: Simone van Geresteyn

Louis Bolk Institute

Secretariat

Hoofdstraat 24

NL-3972 LA Driebergen

Tel. +31 343-523860, Fax -515611

E-mail info@louisbolk.ni

www.suchislife.n

Auskunft: ESCNM

European Society for Classical Natural Medicine

Frau Dr. F. Wilhelmi de Toledo

Postfach 101165

D-88641 Überlingen

Tel. +49 7661 807-0, Fax -889

www.escnm.de

Auskunft: ZÄN

Am Promenadenplatz

D-72250 Freudenstadt

Tel. + 497441 91858-0, Fax -22

E-mail ZAEN-Freudenstadt@t-online.de

www.zaen.org

Auskunft: Deutsch-amerikanische Gesellschaft

für Chiropraktik e.V.

Parkallee 157

D-28209 Bremen

Tel. +49 421347 97-55, Fax -62

Auskunft: Sekretariat SMGP

Keltenstr. 40

CH-8044 Zürich

Tel. +41 125 21-879, Fax -906

E-mail sekretariat-smgp@swissonline.ch

www.smgp.ch

Auskunft: $\mathrm{ZÄN}$

Am Promenadenplatz

D-72250 Freudenstadt

Tel. + 497441 91858-0, Fax -22

E-mail ZAEN-Freudenstadt@t-online.de

www.zaen.org 